\title{
OCIO Y TURISMO MILLENNIAL: EL FENÓMENO DE LAS SALAS DE ESCAPE
}

\author{
Arsenio Villar Lama
}

Universidad de Sevilla

\section{RESUMEN}

El escapismo en vivo es una tendencia de ocio que viene experimentando un crecimiento extraordinario en los últimos años. El objetivo del presente artículo es definir, caracterizar y analizar la evolución de esta actividad, así como evaluar su importancia y su potencial recreativo y turístico en España. Ante la insuficiencia de datos y literatura científica al respecto, hemos recurrido a fuentes de información directa como encuestas y entrevistas, así como a otras menos convencionales como blogs, portales de opinión y otros contenidos generados por usuarios (UGC). Los resultados son reveladores y, entre otros, retratan que este sector emergente - formado por microempresas y estrechamente ligado al colectivo millennial - posee una creciente vinculación con el turismo. Al ser un negocio sin posibilidad de repetición, su éxito entre la población local se traduce en la reducción de la clientela potencial más cercana; así, la captación de la población flotante, estudiantes de larga estancia o turistas, se convierte en una de las principales estrategias de la empresa.

Palabras clave: salas de escape, turismo millennial, user-generated content (UGC), actividades creativas, España.

\section{Millennial leisure and tourism: The rise of Escape Rooms}

\section{ABSTRACT}

The live escape games sector is a leisure trend that has undergone extraordinary growth in the last three years. The aim of this work is to define and characterize the phenomenon, study its evolution and distribution in Spain, and evaluate its leisure and tourism potential.

Recibido: 9 de marzo de 2017

Devuelto para su revisión: 19 de mayo de 2017

Aceptado: 12 de junio de 2017

Departamento de Geografía Física y Análisis Geográfico Regional. Universidad de Sevilla. C/ Doña María de Padilla, s/n. 41004 SEVILLA (España).E-mail: arsenio@us.es 
Given the lack of specific literature or data regarding this topic, it became necessary to generate new and direct information via interviews and surveys and to use less conventional information from user-generated content (UGC) such as blogs and reviews websites. The results are revealing and, among others, show that this emerging sector - formed by microenterprises and closely linked to the millennial generation - has an increasing link with tourism. As offers a service without repetition, capture of local customers is equivalent to reduction of potential users, becoming the attraction of floating population, as the tourists, a key strategy.

Keywords: escape rooms, millennial tourism, user-generated content (UGC), creative activities, Spain.

\section{INTRODUCCIÓN}

El escapismo en vivo es la actividad de ocio más valorada en los principales destinos turísticos europeos. Las salas de escape (o escape rooms, en inglés) han colonizado los primeros puestos de satisfacción de la demanda, según el principal portal de opinión turística TripAdvisor (Cuadro 1); puede comprobarse como en las principales ciudades europeas, las actividades más valoradas se corresponden con este segmento.

\section{Cuadro 1}

\section{ACTIVIDADES DE DIVERSIÓN Y ENTRETENIMIENTO MÁS VALORADAS POR LOS USUARIOS (SE=SALA DE ESCAPE)}

\begin{tabular}{|c|c|c|c|c|c|c|c|c|c|c|c|}
\hline CIUDAD & $1^{\circ}$ & $2^{\circ}$ & $3^{\circ}$ & $4^{\circ}$ & $5^{\circ}$ & $6^{\circ}$ & $7^{\circ}$ & $8^{\circ}$ & $9^{\circ}$ & $10^{\circ}$ & TOTAL \\
\hline PARÍS & SE & SE & SE & SE & SE & SE & SE & SE & SE & SE & $10 / 10$ \\
\hline BERLÍN & SE & SE & SE & SE & SE & SE & SE & SE & SE & SE & $10 / 10$ \\
\hline BARCELONA & SE & SE & SE & $\begin{array}{l}\text { Taller } \\
\text { artesanía }\end{array}$ & SE & SE & SE & SE & SE & SE & $9 / 10$ \\
\hline MADRID & $\begin{array}{l}\text { Estadio } \\
\text { Bernabéu }\end{array}$ & SE & SE & SE & SE & SE & Segway & SE & SE & SE & $8 / 10$ \\
\hline BUDAPEST & SE & $\begin{array}{l}\text { Galería } \\
\text { Tiro }\end{array}$ & SE & SE & SE & Exhibition & SE & SE & SE & SE & $8 / 10$ \\
\hline LONDRES & SE & SE & SE & $\begin{array}{l}\text { Regent's } \\
\text { Park }\end{array}$ & SE & SE & SE & $\begin{array}{l}\text { All } \\
\text { Eng } \\
\text { Tennis }\end{array}$ & SE & SE & $8 / 10$ \\
\hline
\end{tabular}

Fuente: elaboración propia a partir de TripAdvisor (junio de 2016).

Esta actividad consiste en un juego en equipo cuyo objetivo es escapar de una habitación dentro de un tiempo dado, generalmente 60 minutos. El reto suele ir más allá de salir de la sala e incluye una historia con un objetivo concreto: encontrar un tesoro, robar una joya, hallar una prueba clave o desactivar alguna amenaza terrorista programada (bomba atómica, ataque químico), etc.

La dinámica del juego se inicia con la entrada en la habitación. La estancia está llena de objetos y pistas por descubrir. Algunos elementos son accesorios y, por tanto, resulta 
esencial ir discerniendo entre lo ornamental y las pistas verdaderas. Se van resolviendo acertijos y recolectando llaves, objetos o datos que van abriendo cajas fuertes, maletines, cajones, armarios o incluso puertas que dan entrada a otros inesperados habitáculos. En todo momento hay un indicador de cuenta atrás que añade más emoción al reto. A veces el juego incluye la participación de algún actor, pero este hecho es muy minoritario. Los ambientes son muy diversos: una celda, un apartamento, un almacén, un banco, un vagón de tren, un búnker, un manicomio, un laboratorio, etc. A veces recrean otros periodos históricos, por ejemplo, un refugio pirata, una pirámide egipcia o un salón medieval. La ambientación de terror también es recurrente. En algunas ocasiones la temática tiene relación con el ámbito o la ciudad donde se ubica.

Aunque tiene otros precedentes - como veremos más adelante - esta tendencia nace en Budapest en 2012 y se ha extendido de forma vertiginosa por todo el mundo. En España se contabilizan unas 275 empresas (septiembre de 2017), cada vez son más los adeptos al escapismo y el tema ha sido recogido por los medios de forma recurrente ${ }^{1}$. La rápida expansión del fenómeno y la práctica inexistencia de estudios previos al respecto justifican la presente investigación desde las ciencias sociales y, concretamente, desde la geografía del ocio y el turismo.

Frente a una literatura específica prácticamente inexistente en términos académicos y científicos, sí queremos recoger dos corrientes de investigación que contextualizan este fenómeno del escapismo en vivo: el boom del turismo urbano a escala global - entorno principal de estos negocios - y la emergencia de una nueva clase joven y/o creativa en las principales ciudades o destinos.

En relación con la primera, el Global Destination City Index (MasterCard, varios años) confirma que los destinos urbanos son los grandes centroides del turismo global y crecen por encima de los espacios vacacionales litorales, antaño epicentros casi exclusivos del desarrollo turístico. Estos datos a escala internacional se confirman en las agencias nacionales y están alimentando un interesante debate en la comunidad científica, especialmente en torno a los riesgos que conlleva en el plano territorial: masificación provocada por la propia afluencia turística —en ocasiones ya denominada "invasión" (Mondelo, 2017) —, banalización de la imagen tradicional y el impacto paisajístico provocado por señales o muebles urbanos orientados a la actividad (Santos et al, 2016), tematización de los centros históricos y proceso de gentrificación (Serrano, 2013; Fuller y Michel, 2014). Y es que, tal y como recoge Hosteltur (2015) en su interpretación de los citados informes, un éxito mal gestionado puede convertirse en un arma de doble filo desde el punto de vista económico y social para las ciudades.

En España, esta concepción del turismo urbano como turismo masivo viene reflejándose en las principales urbes turísticas como Barcelona, Madrid, Sevilla, Palma, Valencia, Málaga o Granada, pero también en otras ciudades secundarias por debajo del millón de pernoctaciones (Santiago de Compostela, Bilbao, Tarragona, Córdoba...)

En segundo lugar, y en cierta medida frente a la estandarización derivada de este boom turístico, nace precisamente en estas mismas ciudades una nueva clase creativa, gene-

1 La Vanguardia (21/8/2015, 17/5/2016, 15/2/2017), Diario ABC (28/4/2016), Europa Press (31/1/2017), Expansión (15/2/2017), entre otros (véase en las referencias al final del documento). 
ralmente joven, vinculada a la cultura, el ocio y el turismo. Y es que frente a la referida banalización o producción seriada del patrimonio cultural (Harvey, 1989), surgen nuevas formas de entender la relación cultura-turismo al calor de nuevas experiencias turísticas donde la creatividad es el elemento central (Richards y Wilson, 2006; Padilla, 2016). En ocasiones, este oferta creativa se traduce territorialmente en "barrios o lugares creativos" (García et al., 2016; Sánchez et al., 2017), con pequeñas galerías de arte, talleres de artesanía, espacios multifuncionales y co-working, hostelería y otros pequeños negocios como, por ejemplo, salas de escape.

El objetivo del presente artículo es definir, caracterizar y analizar la evolución de esta actividad, así como evaluar su importancia y su potencial recreativo y turístico en España. Para alcanzar este objetivo el documento se estructura de la siguiente manera: en primer lugar se realiza una aproximación conceptual a la actividad, a sus antecedentes y a su marco de referencia (nuevas tendencias recreativas y colectivo millennial). A continuación, hemos hecho un recorrido por el proceso de implantación del fenómeno, especialmente en España. En tercer lugar hemos analizado las características de la oferta y de la demanda, así como una valoración sobre el potencial de las escape rooms como segmento turístico. Concretamente, trataremos de averiguar si esta actividad puede motivar flujos turísticos y, en ese caso, aproximarnos al perfil del viajero. Finalmente, se presentan las conclusiones finales de la investigación.

\section{CONTEXTO}

\subsection{Orígenes de la actividad}

Los precedentes del escapismo en vivo deben buscarse en dos "ambientes" (Nicholson, 2015): en su relación aparente con otras manifestaciones anteriores, de un lado, y en la motivación inicial de los empresarios que han impulsado este fenómeno, de otro. Mediante una encuesta de rango internacional elaborada por el citado autor, se alude a 6 antecedentes del escapismo en vivo: los juegos de rol, las aventuras recreadas en la calle (gymkanas), el teatro interactivo y, principalmente, las aventuras gráficas, el cine y los juegos de escape online.

En el ámbito de los videojuegos, el subgénero de la aventura gráfica o la ficción interactiva conduce al jugador a interactuar con objetos y personajes para ir resolviendo pistas y misterios que le permiten avanzar en la historia. Tiene su origen en "Colossal Cave Adventure", un juego ideado por el programador Will Crowther en 1976 que supone "el inicio de este género narrativo donde converge literatura e informática" (Ruiz, 2007). Se trata de una "aventura conversacional" donde no existe un aparato gráfico, sino una secuencia lógica de instrucciones y decisiones por escrito (Figura 1). A medida que la informática evoluciona, los años ochenta incorporan gráficos, primero estáticos (Mystery House en 1980) y posteriormente dinámicos. En los noventa este género conoce su mayor desarrollo. Las principales firmas serán Sierra y Lucas Arts (fundada por George Lucas), con títulos como King's Quest, Leisure Harry, Maniac Mansion, Indiana Jones o Monkey’s Island. En España destaca la desarrolladora de videojuegos Péndulo Studios, fundada en 1994. 


\section{Figura 1 \\ CAPTURA DE COLOSSAL CAVE ADVENTURE}

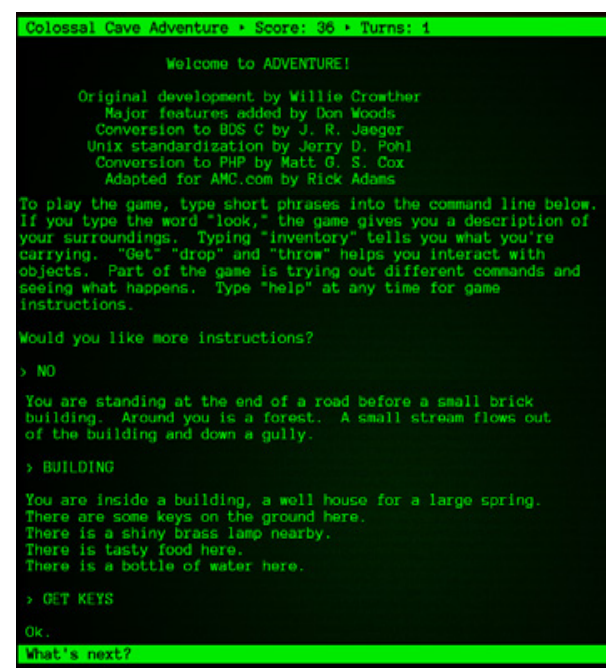

Fuente: Portal de AMC (2017).

Mientras que en los videojuegos existe todo un género vinculado con el escapismo en vivo, los precedentes en el cine son más concretos. Junto a la saga de Indiana Jones de los años ochenta y noventa, la película Cube (1997) y la saga Saw (2004-2010), son consideradas como los principales motores cinematográficos del fenómeno (Nicholson, 2015). El largomentraje español La habitación de Fermat (2007) es la aproximación más parecida a esta temática ${ }^{2}$

Con la generalización de Internet en las primeras décadas del milenio aparece el precedente más directo de esta actividad. Programadores y empresas de todo el mundo desarrollarán juegos de escape online. En un principio estos pasatiempos se jugaban en el ordenador mediante el ratón (point \& click games); en los últimos años, ante la aparición del teléfono inteligente y la tableta, se juega directamente con el tacto. A diferencia del escapismo en vivo, estas aplicaciones, al igual que los videojuegos, no se juegan en equipo.

Con estos antecedentes virtuales, las escape rooms nacen a finales de la primera década del milenio, en torno a los años 2008-2010. Algunos portales hablan de algunos precedentes en Japón sobre $2008^{3}$, mientras que la mayoría coinciden en ubicar en Budapest el origen de las salas de escape actuales. Estas fuentes coinciden en señalar al húngaro

2 En Cube, un grupo de personas buscan la salida de un sistema de habitáculos en forma de cubo. En Saw, un psicópata atrapa y encierra a una o varias personas a las que otorga una oportunidad de salir, siempre que resuelvan acertijos o desactiven trampas en un tiempo determinado. En La Habitación de Fermat un grupo de personas son invitadas a participar en un enigma matemático en una habitación menguante.

3 Concretamente, se alude a la atracción Prision Break del parque de atracciones Joypolis de Tokio, donde la aventura iba más allá de la resolución de acertijos o enigmas (Escape Room Fanatic, 12/1/2017). 
Attila Gyurkovics ${ }^{4}$ como el precursor del fenómeno en 2011. Aprovechó diversos locales semi-abandonados de la ciudad para fundar Parapark (Figura 2) y con su éxito generó un efecto llamada para otros emergentes empresarios. El fenómeno pronto trascendió su papel recreativo para convertirse en un nuevo atractivo turístico de la capital de Hungría. De hecho, la actividad fue nombrada Producto Turístico del año en 2014 por la Asociación Húngara de Agencias de Turismo Receptivo (Daily News Hungary, 2014).

\section{Figura 2 \\ PARAPARK BUDAPEST, CONSIDERADA LA SALA PIONERA DEL ESCAPISMO EN VIVO}

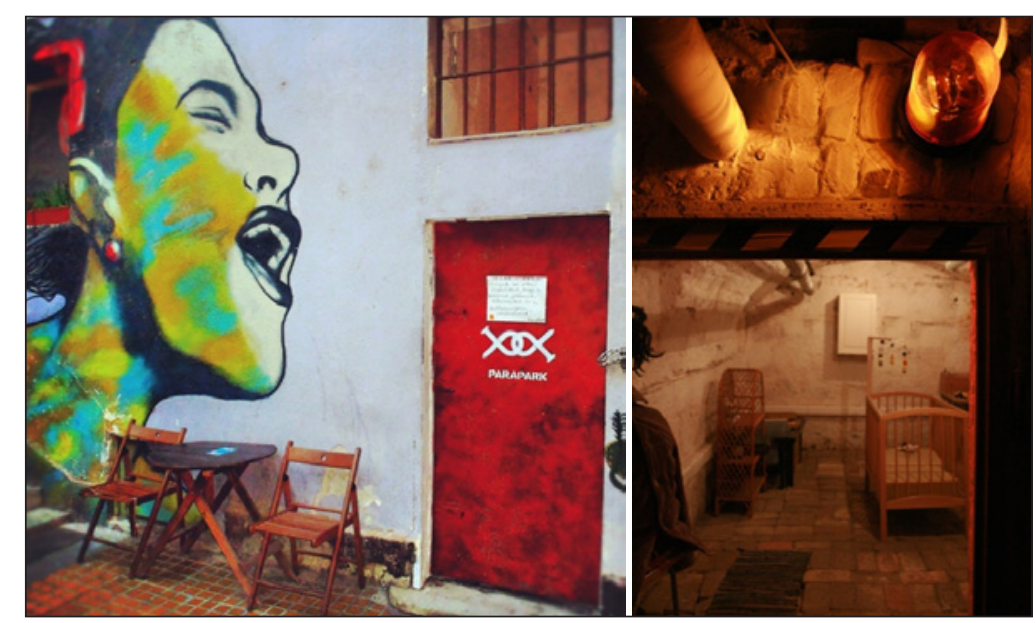

Fuente: Blog de viajes Perth Travellers (2017).

En España la semilla del fenómeno es la franquicia de Parapark en Barcelona en 2013. Algunos de sus primeros jugadores deciden montar sus propias versiones de escapismo (Roomin, Escapa si Puedes y Cronologic). A partir de aquí el fenómeno se extiende, primero en Barcelona y a continuación en el resto de España. En tan solo 3 años el fenómeno se ha popularizado y extendido de forma vertiginosa, como veremos más adelante.

\subsection{Turismo millennial y el impacto de las reviews}

El estilo de vida y las pautas de consumo de los millennials o generación Y - aquellos consumidores nacidos en las dos últimas décadas del siglo XX - están provocando grandes transformaciones en la industria turística (Hosteltur, 2013). Este colectivo ha interiorizado que la globalización y las tecnologías forman parte de su estilo de vida, siendo la interacción virtual el principal mecanismo de relación social y económica. La Organización Mundial del Turismo elabora informes específicos sobre este sector

4 Attila Gyurkovics es el fundador de Parapark, una de las empresas más extendidas en Europa mediante el modelo de franquicia (20 en total, 7 en España). 
joven, afirmando que su peso en la industria turística global no ha parado de crecer en los últimos años: de 190 a 286 billones de dólares (2009-2014), con una proyección a 400 billones en 2020 (World Tourism Organization, 2016).

El perfil turístico de este colectivo se caracteriza, entre otras, por: la búsqueda de un precio competitivo (incluso condicionando fechas y destinos); la reserva sistemática por internet mediante smartphone previa consulta de múltiples fuentes antes de comprar; la importancia que conceden a las opiniones de otros viajeros (blog de viajes y portales de opinión y valoración); un gasto más focalizado en la comunidad local; el valor que dan a la experiencia y, finalmente, por estar conectados a internet en casi toda la cadena de viaje (Hosteltur, 2013; Gustavo, 2013; Cámara, 2015; World Tourism Organization, 2016).

Determinados segmentos del sistema turístico están empezando a adaptarse al cambio provocado por este colectivo - por ejemplo, el segmento aquí analizado, las salas de escape-. En muchos casos, empresarios y clientes pertenecen ambos a esta generación, se entienden y empatizan. Los consumidores tienen una actitud proactiva, son también productores, ya que opinan y generan ideas para mejorar el negocio. Los productores escuchan y tienen una actitud flexible, ya que ellos mismos viajan, opinan y quieren ser escuchados. Las empresas tratan de ser flexibles, implican a todos en el proceso de creación y evolución del producto - en este caso las escape rooms - para hacerlos sentir parte de la marca. Además, se genera una actividad indirecta muy vinculada también a esta generación, los blogs.

$\mathrm{Y}$ es que esta interacción entre consumidores se produce en nuevos canales de contenido generado por usuarios (user-generated content, UGC en adelante), y gracias a la nueva generación de portales en internet que permite la interacción entre iguales, las Web 2.0. (Reactive, 2007). En determinados sectores del turismo - como el sector que nos ocupa - estos canales resultan clave en el éxito del negocio. Por su influencia en el turismo (Lu \& Stepchenkova, 2013) y en el tema abordado, podrían destacarse los siguientes: los portales de valoración de los usuarios (Consumer Reviews), los blogs y las redes sociales. Las consumer reviews (p.e. Tripadvisor, Yelp, Mafengwo, Petit Futé...) suelen ofrecer una valoración numérica de actividades y experiencias, una puntuación media que deriva de las opiniones aportadas por múltiples usuarios. Por su parte, los blogs personales de viaje expresan una valoración - generalmente más detenida y cualitativa - sobre la experiencia general en los destinos turísticos, o sobre segmentos o negocios concretos (alojamientos, gastronomía, actividades de ocio...). Dentro de este colectivo, los denominados "influencers" son aquellos capaces de generar tendencias y condicionar el comportamiento de la demanda. Dada la naturaleza intangible de la experiencia del viaje, los potenciales turistas - sobre todo aquellos que visitan el destino por primera vez- están abrazando estas nuevas formas de compartir el conocimiento mediantes estas comunidades virtuales online (Ricci y Wietsma, 2006; Cox et al., 2009).

El fenómeno de los UGC y su impacto en la industria turística es un tema muy recurrente en las publicaciones científicas de los últimos años; analizándose desde perspectivas variadas (alojamiento, viaje, experiencia, restauración) y utilizado métodos diversos de recolección y tratamiento de datos (Lu \& Stepchenkova, 2013). Munar \& Jacobsen (2014), entre otros, han estudiado las motivaciones sociales y personales que sustentan el compartir la experiencia del viaje; algunas investigaciones se centran en la credibilidad 
de estos canales de opinión (Aye, Au \& Law, 2013) y en su capacidad para decidir el destino (Cox et al, 2009); por su parte, otros estudios estudian la utilidad de los grandes volúmenes de datos para analizar la percepción de la imagen del destino (Marine-Roig y Antón, 2015); así como en canalizar el impacto de los UGCs sobre la gestión turística (Li et al., 2016).

\section{FUENTES Y PROCEDIMIENTO}

Pese al comprobado éxito de esta actividad en diversos portales de opinión turística, su irrupción tan reciente se traduce en una limitada presencia en publicaciones científicas. En el campo de las ciencias sociales, y concretamente en la Geografía del ocio y el turismo, la investigación es inexistente tanto a escala nacional como internacional ${ }^{5}$ (apenas se han encontrado artículos, contribuciones a congresos u otras publicaciones). Este vacío literario nos ha conducido a fuentes de información alternativas: Encuestas y entrevistas dirigidas a empresarios y aficionados, artículos en prensa, blogs y foros específicos de entusiastas del escapismo, así como portales de opinión y satisfacción de la demanda.

La encuesta fue dirigida a las 150 salas de escape de España (de junio a diciembre de 2016), obteniéndose un total de 61 respuestas. Se recibieron réplicas desde 12 Comunidades Autónomas (Cataluña, Madrid, Comunidad Valenciana, Andalucía, Galicia, País Vasco, Baleares, Canarias, Cantabria, Navarra, Castilla y León y La Rioja) y desde las principales provincias (18), un dato muy positivo si se compara con la localización real de la oferta (véase epígrafe 4.1.). Por su parte, se realizaron 7 entrevistas a determinados agentes del sector, 5 empresarios y 2 blogueros con influencia en las tendencias de la demanda ${ }^{6}$.

Desde el punto de vista estadístico, y con el objetivo de aproximarnos al proceso de expansión de esta actividad, se ha tenido acceso al Directorio de Salas de Escape (Escape Room Directory), un registro de Google donde se inscribe toda nueva instalación conocida en cualquier lugar del mundo 7 . Para el caso de España hemos recurrido

5 En el campo de las ciencias de la educación, unos investigadores de la Escuela de Ingeniería de la Universidad Autónoma de Barcelona han publicado recientemente un trabajo sobre la aplicación de un juego de escape en las aulas para promover la motivación y el aprendizaje (Véase Borrego et al., 2016).

6 Los bloggers entrevistados han sido Katia Polenkaya (Escape Room Lover) y Oliver González (Escape Room Fanatic). Las entrevistas tienen la siguiente estructura. En un bloque más general se han realizado una serie de preguntas más cerradas en torno a los siguientes temas: motivación inicial, percepción de saturación del negocio, inducción de economías indirectas, amparo administrativo y protección de derechos de autor. En un segundo bloque, más abierto, se ha hablado de la relación entre esta actividad y el turismo (existencia del turismo de escape, acuerdos con empresas del sector, perfil del turista, visualización del negocio...). La selección de entrevistados se ha basado en un criterio de disparidad locacional (Barcelona, Madrid, Valencia, Sevilla y enclave turístico-costero) y el año de creación de la empresa (2014, 2015 y 2016).

7 Dimensionar este fenómeno a escala global es una complicada tarea, no tanto por la disponibilidad de registros unitarios sino por el grado de actualización de éstos. Para comprobar el grado de verosimilitud de Escape Room Directory se han comparado sus cifras con listados de escape rooms a escala nacional/local. Las cifras son representativas en la mayoría de países o ciudades, aunque tienen cierto desfase temporal, ya que las salas de escape tratan de visualizarse primero en los blogs de alcance nacional, al ser un medio de publicidad más directo. El registro utilizado excluye a China y Japón, por lo que hemos recurrido a sondear otras fuentes alternativas para ponderar el peso de estos países (p.e. EGA China). 
a los blogs de escapismo (EscapeRoom Lover, EscapeRoomFanatic, Cocolacoquette y Taty Hunter), que usualmente incorporan un catálogo elaborado donde aparecen todas las instalaciones ordenadas geográficamente.

El procedimiento de la presente investigación puede subdividirse en cuatro tareas principales. Primeramente se ha compilado y estudiado la información existente sobre el fenómeno para darle un contexto teórico y aproximarnos a la evolución de la actividad (literatura, prensa, foros, registros,...). En segundo lugar, para analizar el sector desde la perspectiva de la oferta y la demanda, se ha llevado a cabo una encuesta dirigida al conjunto de escape-rooms de toda España, así como 7 entrevistas realizadas a diversos agentes del sector (empresarios y blogueros específicos). El acercamiento a la demanda se ha llevado a cabo desde dos perspectivas: de manera indirecta - a través de las preguntas a los empresarios - y mediante el análisis de las valoraciones de usuarios (consumer review), concretamente en TripAdvisor. Para compilar y sistematizar la abundante información contenida en estos portales se ha recurrido a una herramienta de análisis del discurso, concretamente al programa informático Atlas.ti.

\section{RESULTADOS}

\subsection{Dimensión del fenómeno}

Según el aludido documento colaborativo de Google Escape Room Directory, existían a finales de 2017 unas 3.176 empresas en todo el mundo que sumaban 7.721 salas de juego. Un análisis pormenorizado de este registro permite extraer las ideas que se exponen a continuación.

En primer lugar resalta la relativa importancia del modelo de franquicia. Las principales franquiciadoras son las pioneras en el escapismo en vivo y tienen su origen principalmente en Europa, Norteamérica, Rusia y el Sudeste Asiático (Figura 3). La mayoría, aunque centradas en el país de origen, ya poseen franquicias en otras localizaciones. En Rusia destaca Claustrophobia, cuyos creadores han diseñado más de 54 salas de juego que han vendido en 12 países y 34 ciudades (entre ellas Barcelona). La empresa tailandesa Escape Hunt tiene una proyección más internacional y ya se ha adentrado en 27 países y 46 ciudades (Barcelona). En Europa debe señalarse la importancia de Adventure Rooms y la aludida firma húngara Parapark. Adventure Rooms tiene su origen en un proyecto del español Gabriel Palacios ${ }^{8}$, profesor de Física en un Instituto de Berna (Suiza); actualmente posee franquicias en 16 países y 28 ciudades (Madrid, Palma de Mallorca). Por su parte, Parapark está presente en 7 países con 21 franquicias, un tercio de ellas en España (Barcelona, Madrid, Maspalomas, Vigo, La Coruña, Elche y Málaga).

8 Palacios diseñó una secuencia de juegos utilizando experimentos de clase. Los alumnos difundieron la experiencia entre familiares y amigos, al cabo de pocas semanas habían contactado con él unas cincuenta personas que querían disfrutar de su juego. Con su hermano como socio, fundó la empresa. Poco después los periódicos y las televisiones suizas empezaron a informar sobre el proyecto, e inversores extranjeros empezaron a pedir licencias para ofrecer los mismos juegos en otros países (Capital, 2016). 


\section{Figura 3 \\ PRINCIPALES FRANQUICIAS DEL SECTOR Y PRESENCIA EN ESPAÑA (ENERO DE 2017)}

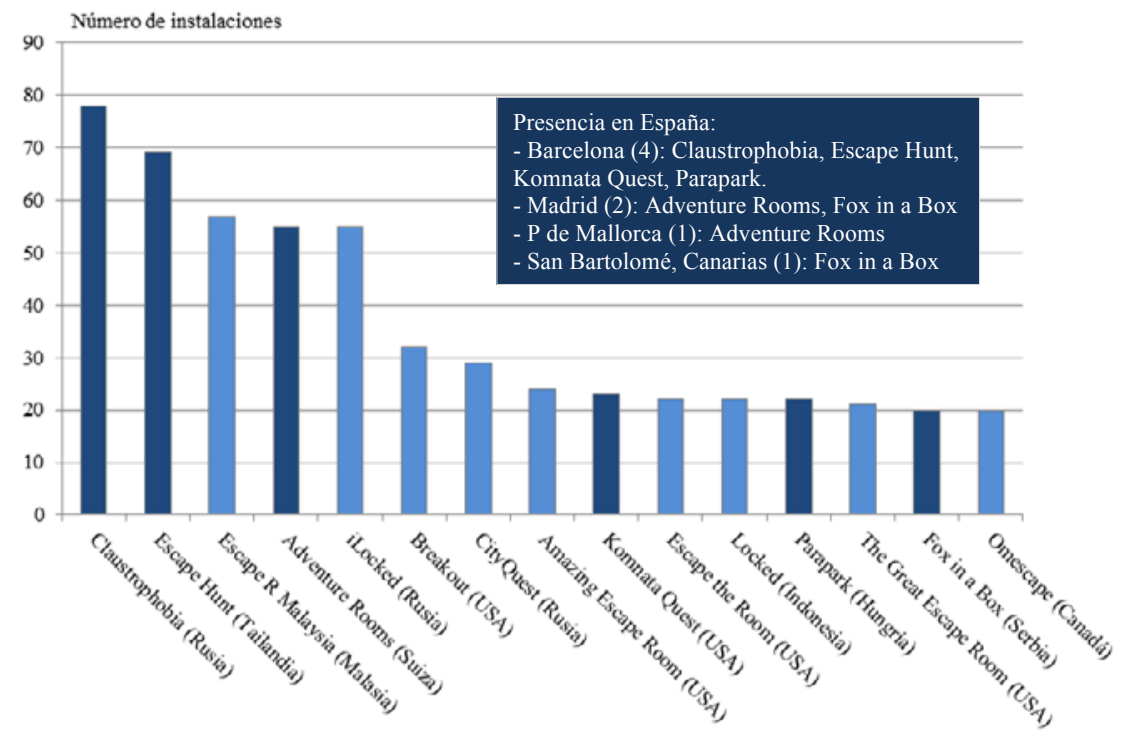

Fuente: elaboración propia a partir de Room Escape Directory.

En segundo lugar, un análisis espacial de los datos informa de la amplia "propagación" del fenómeno por todo el mundo, concretamente en 98 países y 1062 ciudades. Por este orden, Estados Unidos, Canadá, Alemania, España, Malasia y Australia parecen ser los países que han absorbido la mayor parte del negocio (Cuadro 2), a los que habría que sumar China y Japón, fuera de las cifras del registro utilizado. El fenómeno se encuentra muy extendido en Europa: Junto a los países de la Europa Central en torno a Hungría (foco original del fenómeno), destaca su desarrollo en hacia el este (Rusia, Polonia y Rumanía) y en los países mediterráneos, especialmente España.

En España el fenómeno está creciendo de manera vertiginosa. La primera contabilización y mapeo en el verano de 2016 arrojaba un total de 149 instalaciones con 259 salas de juego. Unos diez meses después, en marzo de 2017, estas cifras se doblan y ascienden a 275 y 498 respectivamente ${ }^{9,10,11}$ (Blog Escape Room Lover, 2017). Todas las regiones españolas cuentan con alguna empresa (Figuras 4 y 5) y tan solo 6 provincias no poseen

9 Las cifras del registro mundial no se corresponden con el censo nacional. La correlación del primero respecto al segundo tiene un desfase aproximado de un año. Y es que las escape rooms tratan de visualizarse primero en los registros (blogs) de alcance nacional, ya que es un medio de publicidad más directo.

10 En septiembre de 2017, momento de la primera revisión del presente artículo, el número de empresas había alcanzado llegaba ya 407 y el número de salas de juego a 647, constatando así el extraordinario crecimiento de este segmento. En esta fecha solo 4 provincias seguían ajenas al proceso: Guadalajara, Palencia, Segovia y Soria.

11 En abril de 2018, con la revisión final de esta contribución, se contabilizan 583 empresas y 944 juegos, y sólo Guadalajara y Soria quedan sin instalaciones. 
instalación alguna. Cataluña (97) - y especialmente Barcelona (41) con su área metropolitana (38) - se ha convertido en el epicentro de la actividad ${ }^{12}$. A bastante distancia se sitúan la Comunidad Valenciana (31), Madrid (29) y Andalucía (28). El reciente aumento es especialmente reseñable en estas Comunidades (Cataluña, de 40 a 97; Madrid, de 15 a 29; Andalucía, de 15 a 28), así como en otras más emergentes como Castilla y León (de 8 a 14), Galicia (de 4 a 13), Asturias (de 2 a 10). Si se observa con detalle (Figura 5), se comprobará el nacimiento de un buen número de escape rooms fuera de las capitales provinciales y, especialmente, a lo largo del litoral. El fenómeno no parece haber llegado aún, salvo excepciones, a pequeñas ciudades o ámbitos rurales ${ }^{13}$.

\section{Cuadro 2 \\ ESCAPISMO EN VIVO: PRINCIPALES PAÍSES Y CIUDADES DEL MUNDO (ENERO DE 2017)}

\begin{tabular}{lll}
\hline País & $\mathbf{N}^{\mathbf{0}}$ salas & Ciudades \\
\hline USA & 2672 & Los Ángeles (94), New York (56), Atlanta (48) \\
Canadá & 499 & Toronto (57), Montreal (31) \\
Rusia & 210 & Moscú (84), San Petersburgo (34) \\
R. Unido & 201 & Londres (67) \\
Alemania & 166 & Berlín (21), Frankfurt (21) \\
\hline España & $\mathbf{1 4 9}$ & Barcelona (44), Madrid (21), Valencia (11) \\
\hline Malasia & 142 & Kuala Lumpur (37) \\
Australia & 135 & Melbourne (41), Montreal (31) \\
Polonia & 135 & Varsovia (52) \\
P. Bajos & 106 & Ámsterdam (17) \\
Hungría & 99 & Budapest (80) \\
Rumanía & 97 & Bucarest (69) \\
Italia & 90 & Milán (16) \\
Francia & 84 & Paris (30) \\
Grecia & 76 & Atenas (50) \\
Singapur & 71 & Singapur (71) \\
\hline Otros & 1099 & El Cairo (49), Sao Paulo (38), Viena (33), Estambul, \\
TOTAL & $\mathbf{5 8 3 0}$ & Hong Kong, Praga, Kiev (28) \\
\hline China & 1812 & Singapur (71), Bucarest (69), Londres (67) \\
\hline Japón & No Data & \\
\hline
\end{tabular}

Fuente: elaboración propia a partir de Room Escape Directory y EGA China (2016).

12 A lo largo de 2017 espera inaugurarse en Barcelona el Game Point Center, el centro lúdico de escapismo en vivo más grande del mundo, con 18 salas de escape con temáticas distintas; espera atraer a más de 100.000 personas en los próximos dos años (Torres, 15/02/2017).

13 Can Bruna, en Villafranca del Penedés, se anuncia como la primera sala de escape "en tono rural" de España. 


\section{Figura 4 \\ EVOLUCIÓN DE LA ACTIVIDAD EN ESPAÑA \\ (DE JUNIO DE 2016 A MARZO DE 2017)}

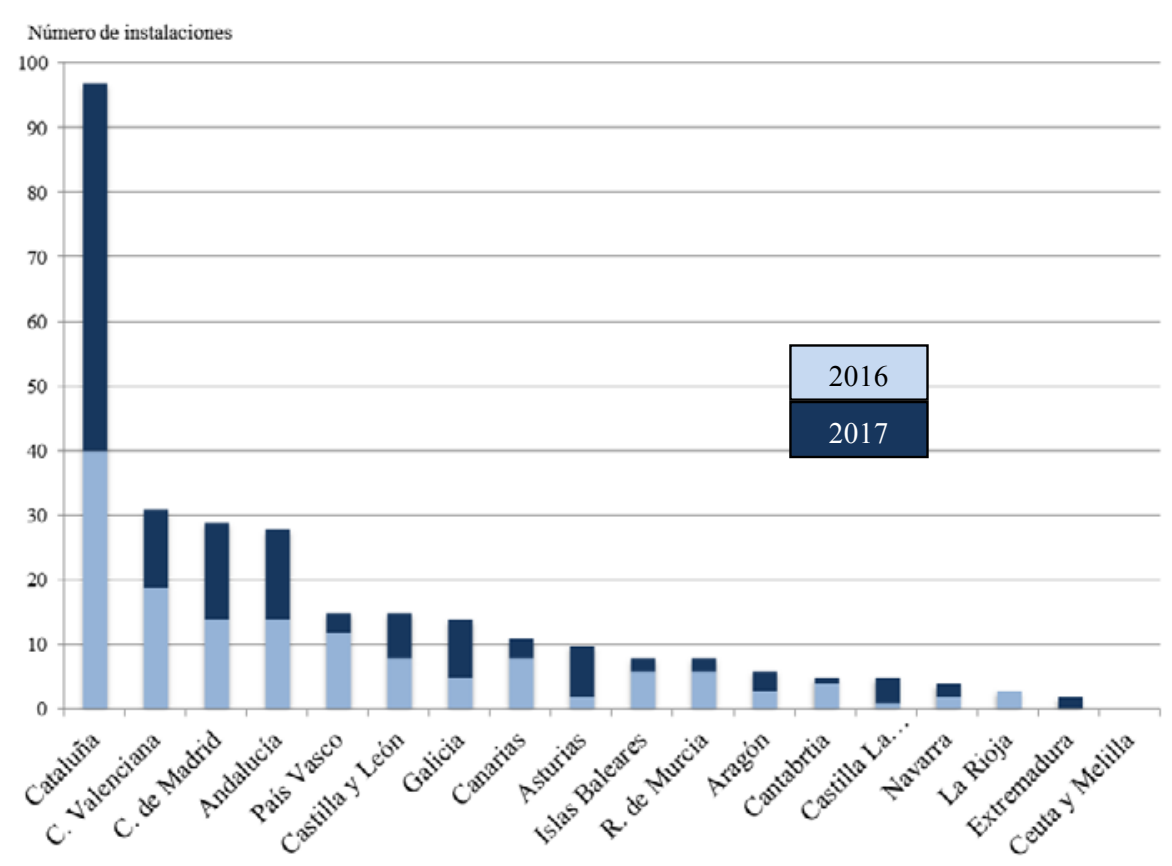

Fuente: elaboración propia a partir de los blogs de referencia.

El desarrollo del sector en España puede dividirse en tres fases. En una primera (2013-2014) se inauguran las primeras salas de escape en Barcelona, Valencia y Madrid. El éxito cosechado por estas primeras instalaciones hace que el fenómeno se multiplique (2014-2015), en primer lugar en estas ciudades y a continuación, de manera más tímida, en otras capitales (Bilbao, Murcia, Santander, Sevilla). Parte de dicha expansión se debe a la génesis de las primeras franquiciadoras españolas (The X Door, Clue Hunter, The Rombo Code $\left.^{14}\right)$. En los dos últimos años (2015-2017) se produce un crecimiento exponencial en el conjunto del país, continuando su difusión entre las principales ciudades, pero también "colonizando" ciudades medias y ámbitos turístico-litorales mediterráneos (Platja d’Áro, Salou, Benidorm, Marbella...).

14 The X Door ha franquiciado ya 9 instalaciones (Madrid, Barcelona, Tarragona, Málaga, Bilbao, La Coruña, Alicante, Lérida) y es la primera empresa española del sector en inaugurar una franquicia en otro país, concretamente en Italia (Milán). Clue Hunter, a partir de su instalación original en Valencia, posee 4 franquicias (Barcelona, Madrid, Murcia y Zaragoza). Finalmente, The Rombo Code es una empresa madrileña que, a partir de su Escape Room en la capital, ha tenido una expansión más limitada y reciente (La Coruña y Sevilla). 


\section{Figura 5 \\ DISTRIBUCIÓN DE LA ACTIVIDAD EN ESPAÑA (DE JUNIO DE 2016 A MARZO DE 2017)}

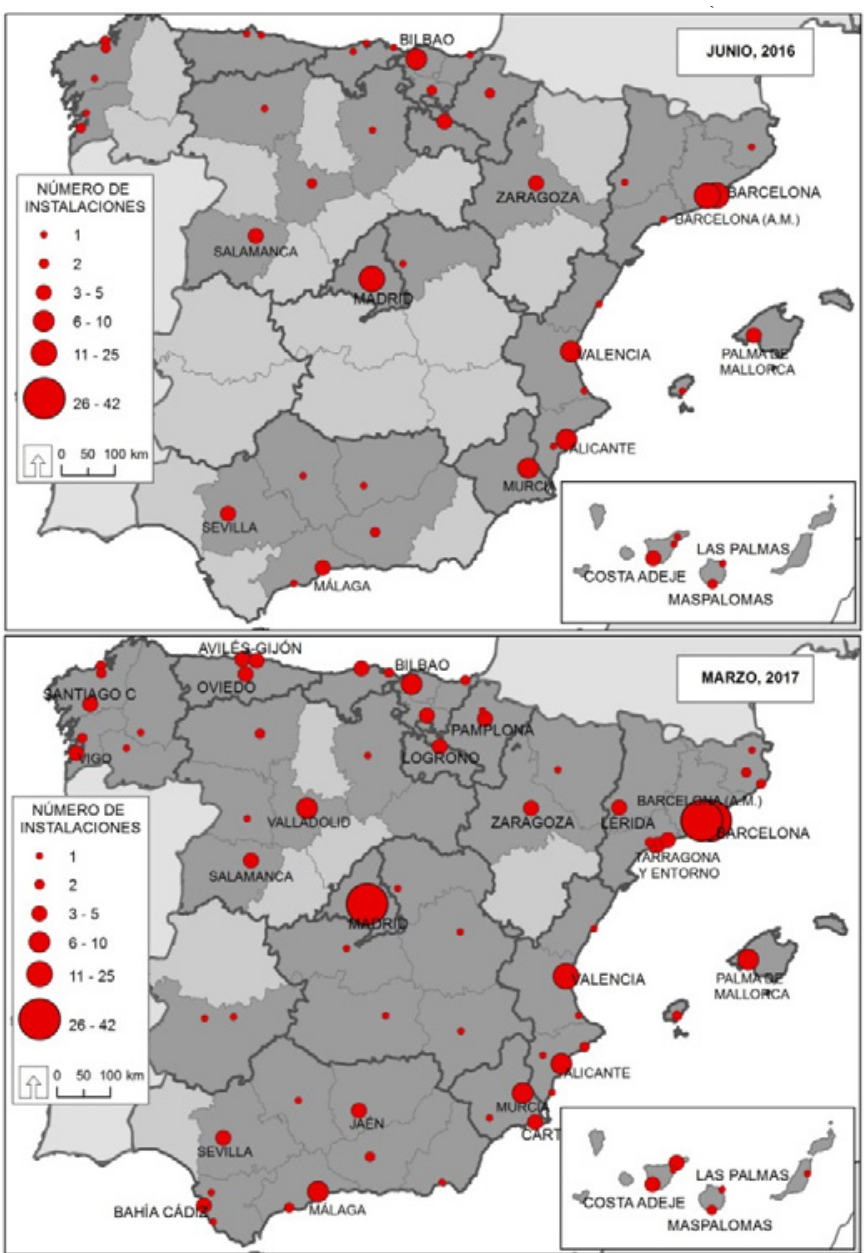

Fuente: elaboración propia. Nota: En gris oscuro las provincias con, al menos, una instalación.

\subsection{Características del negocio en España}

Las características básicas de estas instalaciones - que han sido extraídas de las encuestas y entrevistas realizadas - pueden sintetizarse en las siguientes (Figura 6):

- Predomina la idea original frente a la franquicia (81 frente al 17\%). Parapark (7) y las españolas The XDoor, Clue Hunter y Rombo Code son las franquiciadoras más extendidas. 
- El precio habitual por sesión se sitúa en torno a los 50 euros. Generalmente las instalaciones establecen un precio único, independientemente del número de jugadores. En fechas recientes, especialmente en aquellas ciudades donde hay más competencia (Barcelona, Madrid, Valencia y Bilbao), se buscan nuevas alternativas: precio según el número de participantes, descuentos especiales en días laborables, etc. Por su parte, la inversión inicial implica precios dispares; así, en aquellas salas donde hay una apuesta fuerte por la tecnología (electrónica, láseres...), el precio tiende a aumentar para recuperar a corto o medio plazo la inversión realizada.

- La empresa está conformada por una media de 2,04 socios. Por lo general son unipersonales (39\%) o de dos socios (29\%). En muchos casos se constata la fuerte relación previa (amigos, familia o pareja). La edad media de estos empresarios se sitúa en torno a los 25 y 40 años (96\%), y concretamente entre 30 y 34 (44\%). Ello nos conduce a considerarlo como un negocio fuertemente vinculado al colectivo millennial. En algo más de la mitad de los casos, la sala de escape conforma la única fuente de ingresos del empresario (55,7 frente al 44,3 por ciento).

- Habitualmente los socios trabajan en el local, pero se contrata a un promedio de 2 asalariados. En este caso existe una notable variabilidad. Lo más común es que los propios socios se vuelquen totalmente en el negocio, sin realizar ninguna contratación (sin asalariados 39\%). Por otra parte, algunas empresas, generalmente con un mayor número de salas de juego, contratan a más de 1 empleado $(37 \%)$.

- La inquietud por recuperar la inversión inicial deriva también en una dedicación importante en cuanto a tiempo de apertura durante el día, la semana o el año. La mitad no cierra la instalación por vacaciones (49\%) y una cuarta parte apenas lo hace durante una semana (26\%). Apenas tienen un día de descanso a la semana y, respecto al horario, generalmente las salas ofrecen sesiones en turnos de mañana, tarde e incluso noche.

- Todas las instalaciones tienen página Web traducida a varios idiomas (inglés, 73\%; francés, $11 \%$; italiano, $9 \%$; alemán, $8 \%$ ) y poseen gestores de reserva online y pago telemático. Por lo común, los portales tienen un diseño amable y atractivo. Todo ello es, en parte, reflejo del perfil del empresario, joven y fuertemente vinculado a su negocio.

- La principal debilidad del negocio es el patrón de no-repetición del cliente. El jugador, una vez realizada la actividad, no volverá a menos que se diseñe una nueva sala. Se convierte en difusor de la experiencia en su círculo más cercano, mediante el boca a boca, o al público en general a través de portales de opinión. El buen trato al cliente y la gestión de la imagen de la empresa en estos foros son piezas clave del negocio.

En resumen, el perfil tipo del negocio sería una empresa realizada a partir de una idea original, montada por uno o dos socios de entre 25 y 35 años. Los propios socios gestionan la instalación y no se contrata a personal adicional. La intensidad del trabajo es importante, en la medida que no cierran por vacaciones, apenas tienen un día de descanso semanal y están abiertos en horario de mañana y tarde. Son conscientes de que Internet es la puerta de entrada de sus clientes y, de esta forma, "cuidan" muchísimo sus páginas Web (diseño, traducción a varios idiomas, pago telemático) y atienden sus perfiles en los portales de opinión de la demanda. 


\section{Figura 6 \\ RASGOS BÁSICOS DE LA OFERTA}

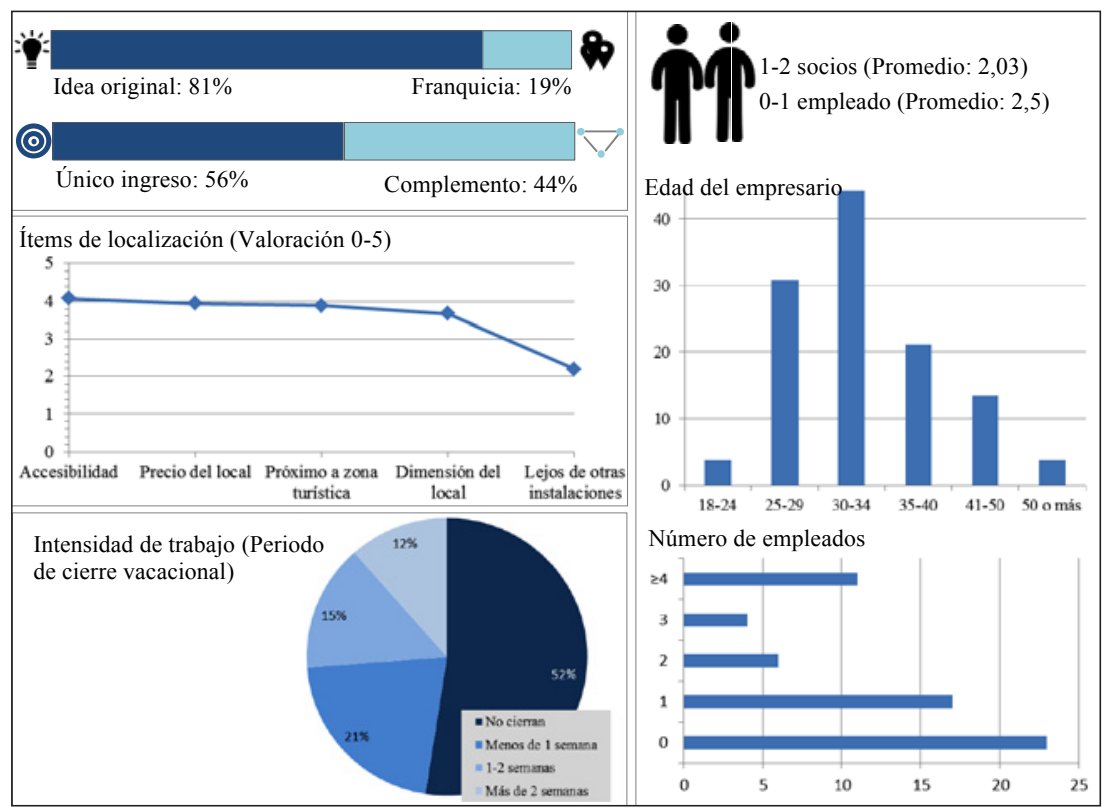

Fuente: elaboración propia.

A partir de las entrevistas realizadas, el encaje legal de estas nuevas empresas no ha sido fácil. En un primer momento, se trataba de un negocio donde los socios se declaraban autónomos y se inscribían en los registros correspondientes como "salas recreativas", "actividades de juegos de azar y apuestas" u "otras actividades recreativas" (categorías CNAE). Ante la dimensión alcanzada por el fenómeno, se espera una categoría propia a corto-medio plazo. En esta línea de la regularización, una de las principales controversias del sector es la protección de los derechos de autor. El colectivo tiene bien asumido la copia de algunos juegos específicos, pero considera poco probable el uso de la idea completa, no tanto por la sanción administrativa — de difícil aplicación por el encaje legal de la actividad - sino por la mala publicidad en la red que podría conllevar.

En relación con la localización, la necesidad de contar con un local de ciertas dimensiones y la limitada capacidad de inversión inicial - factor nuevamente ligado a la edad media del empresario - conduce a la actividad hacia a los ensanches de la ciudad, donde la accesibilidad y el precio son moderados (Figura 7). La periferia ofrece precios más bajos y mayores posibilidades de expansión (nuevas salas de juego); sin embargo, son pocas las empresas que han optado por ubicarse en los bordes de la ciudad. Y es que según la encuesta, los factores más importantes para los administradores son, por ese orden: estar bien comunicado, el precio de compra o alquiler, estar cerca de la zona turística, la dimensión del local y la proximidad de otras salas de escape. Para las instalaciones que se implantan en localidades litorales (Benidorm, Torrevieja, Marbella, Conil, Maspalo- 
mas) la proximidad a los espacios turísticos resulta especialmente relevante. El patrón de localización está muy condicionado por la inversión inicial: Las empresas más humildes suelen ubicarse en calles menos accesibles, mientras que las franquicias y otros proyectos más grandes se sitúan en avenidas, zonas peatonales y/o vías próximas al flujo turístico.

\section{Figura 7 \\ LOCALIZACIÓN DE LAS SALAS DE ESCAPE EN LAS PRINCIPALES CIUDADES DE ESPAÑA}

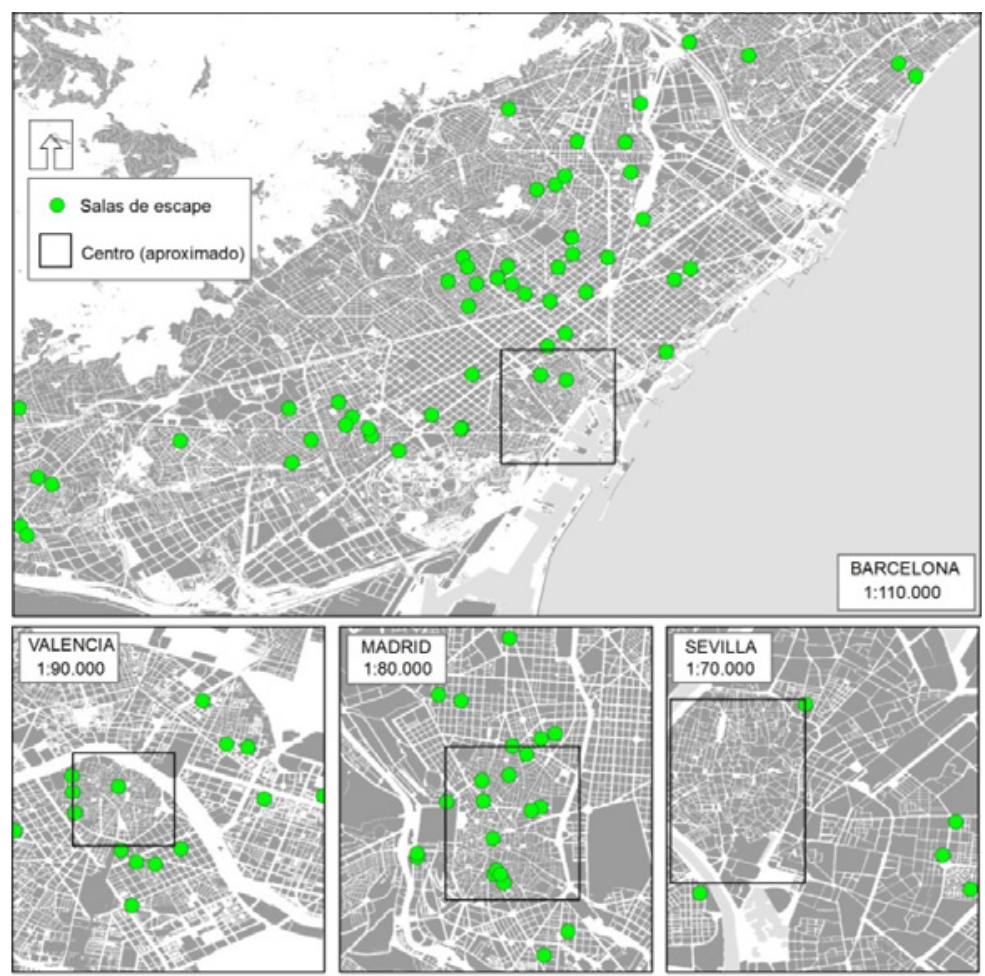

Elaboración propia a partir de los blogs de referencia.

Finalmente, en relación a los rasgos básicos de la demanda, se caracteriza de la siguiente manera (Figura 8): Se trata de gente joven, de entre 21 y 35 años (80\%), que realiza la actividad principalmente con amigos (53\%), y en menor medida con la pareja, la familia y los compañeros de trabajo ${ }^{15}$. Se trata por tanto de un negocio muy ligado a la generación "Y", tanto por los empresarios que abren estas instalaciones como por la edad de la demanda. Los factores de decisión son principalmente la historia del juego (38\%)

15 Rombo Code en Madrid destaca por la captación de empresas que desean hacer jornadas de formación de equipos o team-building. La actividad, que puede ser grabada, permite comprobar a estas compañías los diversos roles de sus empleados (lógica, observación, inteligencia emocional, liderazgo...) 
y las referencias previas (32\%). El precio, a diferencia de la caracterización general de este colectivo turístico, se convierte aquí en un factor subsidiario, hecho que podría relacionarse con una edad media relativamente alta (el grupo predominante es el de 31-35 años). Por su parte, la localización también desempeña un rol secundario: "Si la historia es buena y el juego ha sido recomendado, el desplazamiento es lo de menos" (recogido de las entrevistas realizadas).

\section{Figura 8 \\ RASGOS BÁSICOS DE LA DEMANDA}

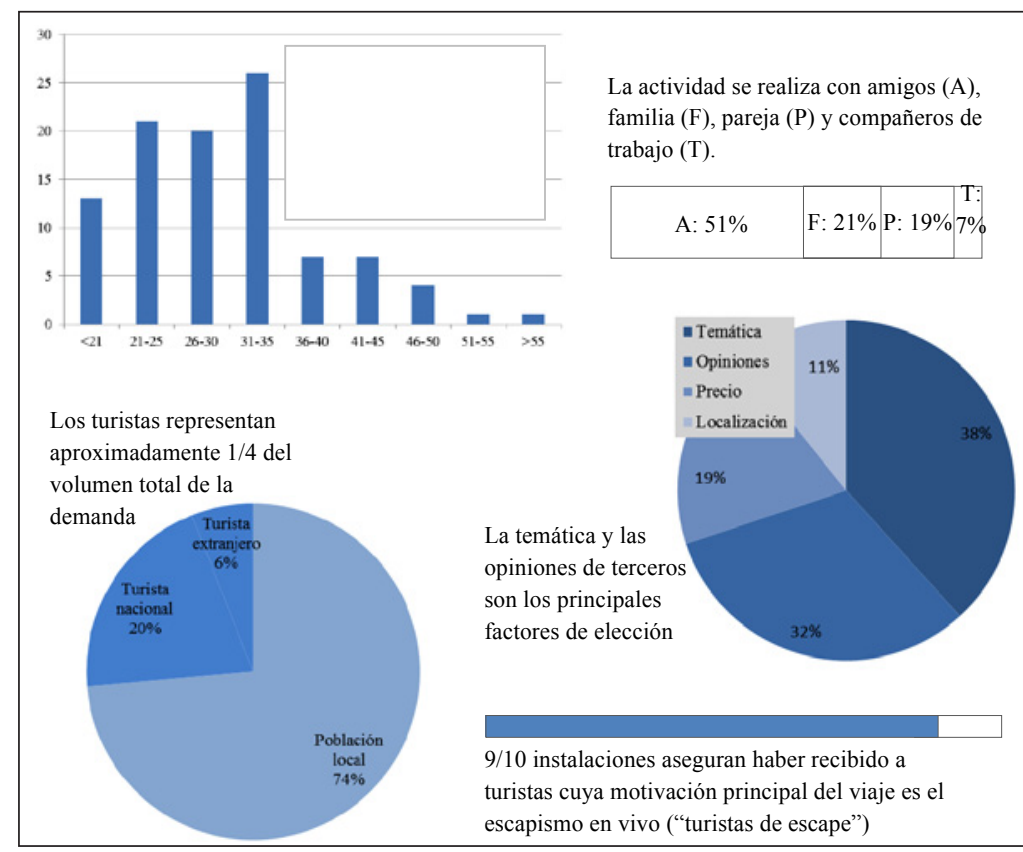

Fuente: elaboración propia y Escape Room Lover (3/3/2017)

\subsection{Importancia como actividad turística}

El escapismo en vivo es una actividad ligada fundamentalmente a la recreación no turística. Según la encuesta, el turismo representa una cuarta parte del volumen total de demanda $(25,9 \%)$. De los turistas, en torno el 26,9 por ciento son extranjeros. Los administradores, no obstante, aseguran que este colectivo es importante $-\mathrm{y}$ lo será aún más en el futuro, especialmente en las principales ciudades - . Conscientes de esa relevancia, el empresario posiciona su instalación en los principales blogs a escala nacional (y en menor medida internacional), gestiona cotidianamente las valoraciones recibidas desde los portales de opinión y se acerca al turista extranjero traduciendo su Web a varios idiomas. Para atender al visitante foráneo, los socios y/o empleados de la instalación dominan el inglés para introducir y guiar el juego (temática, objetivo y posibles pistas puntuales). 
El escapismo en vivo nace como una nueva forma de ocio local pero, según Oliver González, creador del primer blog sobre el tema en España, “aquellos primeros aficionados empiezan a dirigir sus pequeños viajes de fin de semana hacia destinos con salas de escape". Aunque la motivación principal del viaje era otra (cultural, sol y playa o la visita a familiares), la existencia de escape rooms empieza a convertirse en un factor de decisión. Según una encuesta planteada a los usuarios del blog Escape Room Lover, casi la mitad incluyen esta actividad en sus planes de viaje, a veces (33\%) o siempre (14\%). Como se deriva de las encuestas, recientemente se podría hablar de un "turismo de escape", aún minoritario, según el cual "jugar las salas” pasa a ser la motivación principal del viaje ${ }^{16}$.

Solo las ciudades con una oferta suficiente reciben a "turistas de escape"; así, según las entrevistas realizadas, Barcelona, Madrid, Valencia y Bilbao son los destinos de este emergente segmento turístico. En Barcelona se tiene constancia de viajeros internacionales atraídos por la cultura del escapismo que realizan jornadas maratonianas (Picadero Motel, 22/4/2016). Sin embargo, las rutas en coche a lo largo de diversas ciudades con un número más modesto de salas conforman una incipiente alternativa (p.e. en la cornisa cantábrica, en Castilla y León o Andalucía). De hecho, algunas empresas han creado alianzas a través de la creación de una liga de escape rooms, donde se incita al cliente a completarla consiguiendo premios finales o descuentos (p.e. "Ruta Norte" o "Misterios de Castilla").

A pesar de su magnífico posicionamiento en portales de contenido generado por los usuarios (p.e. Tripadvisor), y a pesar de ser considerados los millennials - consumidor principal del producto - como el colectivo de moda en el turismo, las salas de escape aún no están suficientemente reconocidas ni categorizadas en la normativa y en la publicidad institucional. No obstante, el sector ha conseguido posicionarse en guías tradicionales de reconocido prestigio (p.e. Lonely Planet, Le Routard), así como en algunas páginas oficiales de turismo urbano (Visitlondon, Parisinfo, Newyorkcitygo, Budapestguide).

\section{CONCLUSIONES}

En un mundo cada vez más tecnológico y digital las mejores experiencias en vivo y offline florecen. Respecto a los juegos digitales u otras actividades, el escapismo en vivo introduce varias características que explican su fulgurante éxito: la interacción real con objetos, la desconexión con el "mundo exterior", la emoción del momento y la dinámica de grupo. Con el trasfondo del videojuego o el cine, aquí el usuario se convierte en el protagonista de la historia y, lo que es más importante, comparte esa experiencia con iguales (pareja, amigos, familia o compañeros de trabajo). Ello permite sacar a la luz las capacidades de todos los miembros del grupo, desde la lógica y la deducción a la capacidad de observación o el liderazgo (independientemente del nivel de formación de cada jugador). El hecho de apagar el teléfono y estar una hora concentrado en una actividad real y en grupo que genera una buena cantidad de adrenalina conforman los ingredientes

16 Según la encuesta, un $90,4 \%$ de las empresas encuestadas han recibido a este perfil de "turista de escape", definido por parejas o grupos de entre 25 y 35 años que viajan con la motivación principal, aunque no exclusiva, de jugar entre 2 y 5 salas durante su estancia. 
perfectos para lo que Csikszentmihalyi (2008) denomina el "flujo en grupo" - un estado mental donde un conjunto de personas trabajan coordinados bajo un mismo objetivo y están completamente inmersas en la experiencia que realizan - y que, asegura, aporta una buena dosis de felicidad.

La principal debilidad del sector es que ofrecen un servicio sin repetición, razón por la cual el "boca a boca" (real u online) se convierte en el factor clave para el éxito del negocio. El problema es que la captación de clientes locales equivale a la reducción de los futuros usuarios en ese entorno más inmediato. Ahí es donde entra el turismo; a medida que se va agotando la población del lugar como clientes, la captación de turistas (o estudiantes de larga duración como Erasmus) llega a convertirse en la principal estrategia de la empresa. Si esto no es suficiente, y a modo de recomendación, si se observan síntomas de agotamiento del producto $-\mathrm{y}$ teniendo en cuenta el actual contexto de la economía colaborativa y el perfil joven y dinámico de estos empresarios - , las instalaciones de distintas ciudades podrían permutar sus juegos a medio plazo. Ello permitiría renovar el producto con una inversión menor. Otras medidas más puntuales que ya se han implementado se basan en la tematización del juego con eventos concretos (Navidad, Halloween...).

La diversidad de salas se convierte en la principal fortaleza de este sector como segmento turístico. Una vez que los adeptos a la actividad superan los retos de su entorno inmediato se convierten en turistas que desean enfrentarse a nuevos desafíos en otras ciudades. De hecho, esta investigación comprueba la existencia de "turistas de escape" o personas que viajan con la motivación exclusiva o prioritaria de realizar esta actividad varias veces durante el viaje. Las franquicias repiten los mismos juegos y temas en destinos distintos y su excesiva proliferación podría considerarse como la principal amenaza de esta actividad como práctica turística.

El escapismo en vivo encaja bien por lo general en las líneas actuales de desarrollo turístico, como son la sostenibilidad, el valor de la experiencia y el contacto y la promoción de la cultura local: fortalece y diversifica la economía, implica emprendimiento y empleo (especialmente entre los más jóvenes), amplían el espacio turístico, hay un diálogo entre el local (creador del juego) y el visitante (jugador), y, finalmente, se proporciona una experiencia única, en el marco del turismo creativo. Sin embargo, la creciente difusión de las franquicias y la escasa conexión de las tramas con la cultura o el patrimonio local son atisbos de estandarización, por lo que deberían fortalecerse historias, tramas o leyendas vinculadas con la región o la ciudad donde se ubican.

\section{REFERENCIAS BIBLIOGRÁFICAS}

AYEH, J., AU, N. y LAW, R. (2013): "Do We Believe in TripAdvisor? Examining Credibility Perceptions and Online Travelers attitude toward Using User-Generated Content", Journal of Travel Research, $\mathrm{n}^{\circ} 52$ (4), pp. 437-452.

BENÍTEZ, C., BLANCO, A. y IRISARRI, J.M. (Prods.), PIEDRAHITA, L. y SOPEÑA, R. (Dirs.) (2007): La habitación de Fermat [largometraje]. España: Nostro Films, Bocaboca y Manga Films.

BRUNTON, C., MEH, M. y ORR, B. (Prods.) y NATALI, V. (Dir.) (1997): Cube [largometraje]. Canadá, Viacom. 
BORREGO, C., FERNÁNDEZ C., ROBLES S. y BLANES I. (2016): “Room Escape en las aulas: actividades de juegos de escape para facilitar la motivación y el aprendizaje de las ciencias de la computación", Revista del Congrés Internacional de Docencia $i$ Innovació, $\mathrm{n}^{\mathrm{o}} 3$, pp. 1-7.

CAMARA, F. (2015): Millennials: la generaçó que revoluciona el turismo (Trabajo Fin de Grado). Universidad de Lleida, Facultad de Derecho y Economía. Lérida.

COX C., BURGESS S., SELliTTO C. y BUULTJENS J. (2009): “The Role of UserGenerated Content in Tourists' Travel Planning Behavior", Journal of Hospitality Marketing \& Management, no. 18:8, pp. 743-764.

CSIKSZENTMIHALYI, M. (2008): Fluir (Flow), una psicología de la felicidad. 13a edición. Editorial Kairós. Barcelona, 448 pp.

FÜLLER, H. y MICHEL, B. (2014): “ 'Stop Being a Tourist!' New Dynamics of Urban Tourism in Berlin-Kreuzberg", International Journal of Urban and Regional Research, $n^{\circ} 38$, pp. 1304-1318.

GARCÍA, A., FERNÁNDEZ, V., CARAVACA, I. y GONZÁLEZ. G. (2016): “Actividades creativas, transformaciones urbanas y paisajes emergentes. El caso del casco norte de Sevilla", Documents d'Anàlisi Geográphica, no 62 (1), pp. 27-54.

GUSTAVO N. (2013): "Marketing Management Trends in Tourism and Hospitality Industry: Facing the 21st Century environment", International Journal of Marketing Studies, $\mathrm{n}^{\mathrm{o}} 5$, pp. 13-25.

HARVEY, D. (1989): The Condition of Postmodernity. Basil Blackwell. Oxford, 392 pp.

HOFFMAN, G., BURG, M., KOULES, O. (Prods.), HACK, L., LYNN, D. y WAN, J. (Dirs.) (2004-2010): Saw [saga de largometrajes]. Estados Unidos: Lionsgate.

HOSTELTUR (2013): "Millennials, el target de moda del turismo". Revista mensual: Noviembre de 2013.

HOSTELTUR (2015): "El turismo urbano vive un boom a escala global". Revista mensual: Octubre de 2015. Disponible en https://www.hosteltur.com/113130_turismo-urbano-viveboom-escala-global.html

LU, W. y STEPCHENKOVA, S. (2015): "User-Generated Content as a Research Mode in Tourism and Hospitality Applications: Topics, Methods, and Software", Journal of Hospitality Marketing \& Management, $\mathrm{n}^{\circ}$ 24, pp. 119-154.

MARINE-ROIG E. y ANTÓN, S. (2015): “Tourism analytics with massive user-generated content: A case study of Barcelona”, Journal of Destination Marketing \& Management, $\mathrm{n}^{\circ} 4$, pp. 162-172.

MASTERDCARD (varios años): Global Destination Cities Index. Disponible en https:// insights.mastercard.com/topics/economic-outlook/

MUNAR A.M. y JACOBSEN, J.K (2014): "Motivations for sharing tourism experiences through social media", Tourism Management, $\mathrm{n}^{\circ}$ 43, pp. 46-54.

NICHOLSON, S. (2015): Peeking behind the locked door: A survey of escape room facilities (Documento auto-publicado). Disponible en http://scottnicholson.com/pubs/ erfacwhite.pdf

PADILLA, Y. (2016): Metamorfosis de una invención. Turismo y clase creativa: el caso de la Costa del Sol en el mediterráneo español (Tesis Doctoral). Servicio de Publicaciones y divulgación científica de la Universidad de Málaga. 343 pp. 
REACTIVE (2007): Web 2.0 for the tourism \& travel industry. Reactive. Melbourne.

RICCI, F. y WIETSMA, R. (2006): "Product reviews in travel decision making", en Information and communication technologies in tourism (International Conference in Lausanne). Lausanne, pp. 296-307.

RICHARDS, G. y WILSON, J. (2006): “Developing Creativity in Tourist Experiences: A Solution to The Serial Reproduction of Culture?", Tourism Management, $\mathrm{n}^{\circ} 27$ (6), pp. 1209-1223.

RUIZ-NÚÑNEZ, J. (2007): “El origen de las aventuras gráficas. La ficción interactiva”, en CDC Cuadernos de Comunicación, $\mathrm{n}^{\circ}$ 1, pp. 154-163.

SÁNCHEZ, J.L., NICOLÁS, A., ALONSO, J.L. y MORO L. (2017): "Regeneración urbana, innovación social y prácticas económicas alternativas en ciudades medias: el Barrio del Oeste (Salamanca)", Ería, no 1 (nueva época), 67-82.

SANTOS, E., FERNÁNDEZ, A. y ALVARADO, O. (2016): “La incorporación del paisaje a la planificación turística. Análisis de la estrategia de turismo sostenible de Andalucía”, Cuadernos de Turismo, no 37, pp. 175-202.

SERRANO, A.M. (2013): “Gentrificación. globalización, turismo y patrimonio”, Cambios y permanencias, $\mathrm{n}^{\circ} 4$, anexos.

WORLD TOURISM ORGANIZATION (2016): Affiliate Members Global Reports, Volume Thirteen - The Power of Youth Travel. UNWT. Madrid, 58 pp.

\section{OTRAS REFERENCIAS}

AGUILAR C. (8/2/2017): "Escape Room en Sevilla: el reto de salir de una habitación en menos de una hora". Diario $A B C$. Disponible en http://sevilla.abc.es/sevilla/seviescape-room-sevilla-reto-salir-habitacion-menos-hora-201702082316_noticia.html

AMC Networks (20/03/2017): "Colossal Cave Adventure". Disponible en http://www. amc.com/shows/halt-and-catch-fire/exclusives/colossal-cave-adventure

CAPITAL (Magacín) (2016): “Adventure Rooms: Llegó el momento de escapar”. Disponible en http://www.capital.es/2016/04/25/

DAILY NEWS HUNGARY (27/12/2014): "Room Escape Game is the tourist product of the year". Daily News Hungary. Disponible en https://dailynewshungary.com/roomescape-game-is-the-tourist-product-of-the-year/

DELGADO, A. (28/4/2016): "Escape Room: cómo salir de una habitación en 60 minutos". Diario ABC. Disponible en http://www.abc.es/plan-b/relajarse/abci-escape-roomfiebre-juegos-escapismo-madrid-201604282216_noticia.html

EGA China: Disponible en http://www.chinaega.com/

ESCAPE ROOM LOVER (30/6/2016 y 3/3/2017): El Directorio más completo de Escape Rooms [Blog de escapismo]. Disponible en http://www.escaperoomlover.com

FERNÁNDEZ, P. (31/1/2017): “¿Por qué deberías probar una Room Escape (o zona de escape)?". Europa Press. Disponible en http://www.europapress.es/comunicados/sociedad-00909/noticia-comunicado-deberias-probar-room-escape-zonaescape-20170131155900.html

GONZÁLEZ, O. (12/1/2017): Turismo de escape, ¿qué es? [Blog de escapismo Escape Room Fanatic]. Disponible en http://escaperoomfanatic.blogspot.com.es/ 
LONELY PLANET (2016): “A race against the clock: Budapest's live escape games”. Disponible en https:/www.lonelyplanet.com/hungary/budapest/travel-tips-and-articles/arace-against-the-clock-budapests-live-escape-games.

MONDELO, V. (26/6/2017): "No es turismo, es una invasión”. Diario El Mundo. Disponible en http://www.elmundo.es/economia/2017/06/26/594d08b322601d577e8b461b. html

MONTILLA, R. (17/5/2016): "Más de 100 empresas ofrecen juegos de escape en Barcelona". La Vanguardia. Disponible en http://www.lavanguardia.com/local/barcelona/20160517/401839505112/mas-de-cien-empresas-ofrecen-juegos-de-escape-enbarcelona.html

PARTAL, T. (21/8/2015): "Room Escape: Una nueva forma de jugar”. La Vanguardia. Disponible en http://www.lavanguardia.com/ocio/20150821/54435903414/room-escape. html

PERTH TRAVELLERS (16/10/2012): “The most fun you can have whilst trapped in a tiny room. Budapest, Hungary" [Blog de viajes]. Disponible en https://2perthtravellers. wordpress.com/

PICADERO MOTEL (22/4/2016): "Turismo de Room Escape" [Blog de escapismo]. Disponible en http://www.picaderomotel.com/blog/turismo-de-room-escape/

PRIETO A. (15/2/2017): "Una empresa rusa invierte 15 millones en un centro de juegos de escape en Port Fòrum”. Expansión. Disponible en http://www.expansion.com/catal unya/2017/02/15/58a44891468aebf82f8b4611.html

RODRIGO, E. (22/2/2017): Página principal [Blog de escapismo Cocolacoquette]. Disponible en http://www.cocolacoquette.com/escape-en-vivo/

TATY HUNTER (7/7/2017): "Rutas de salas de escape" [Blog de escapismo]. Disponible en http://www.tatyhunter.com/

TORRES, J. (15/2/2017): “La instalación de 'escape room’ más grande de Europa aterriza en Barcelona”. La Vanguardia. Disponible en http://www.lavanguardia.com/local/ barcelones-nord/20170215/4247507351/instalacion-escape-room-mas-grande-europaaterriza-barcelona-sant-adria-port-forum.html 